\title{
ON EQUILIBRIUM POINTS OF LOGARITHMIC AND NEWTONIAN POTENTIALS
}

\author{
J. CLUNIE, A. EREMENKO AND J. ROSSI
}

\begin{abstract}
Let $f(z)=\sum_{j=1}^{\infty} a_{j} /\left(z-z_{j}\right)$, where $z_{j} \neq 0$ and $\sum_{j-1}^{\infty}\left|a_{j}\right| /\left|z_{j}\right|<\infty$. Then $f$ can be realized as the complex conjugate of the gradient of a logarithmic potential or, for integral $a_{j}$, as the logarithmic derivative of a meromorphic function. We investigate conditions on $a_{j}$ and $z$, that guarantee that $f$ has zeros. In the potential theoretic setting, this asks whether certain logarithmic potentials with discrete mass distribution have equilibrium points.
\end{abstract}

\section{Introduction}

In this paper we will discuss the following problem. Consider the meromorphic function

$$
f(z)=\sum_{j=1}^{\infty} \frac{a_{j}}{z-z_{j}}, \quad \sum_{j=1}^{\infty} \frac{\left|a_{j}\right|}{\left|z_{j}\right|}<\infty
$$

where $z_{j} \neq 0$. Under what conditions on $a_{j}$ and $z_{j}$ can one guarantee that $f$ has zeros?

This question is an extended version of a problem proposed by L. Rubel [2, Problem 7.78]. We shall suppose that $a_{j} \in \mathbb{R}$. Then the problem has the following physical interpretation: if we imagine particles (really charged wires perpendicular to the complex plane) placed at each point $z_{j}$ and having the charges $a_{j}$ then these generate a logarithmic potential given by

$$
u(z)=\sum_{j=1}^{\infty} a_{j} \log \left|1-\frac{z}{z_{j}}\right| .
$$

The gradient of this potential is the complex conjugate of $f$. Thus the question is whether such a field must always have an equilibrium point, that is a point upon which a free electron (or wire) once placed would remain.

This question and the corresponding problem in $\mathbb{R}^{n}, n \geqslant 3$ stimulate an interesting area of research-distribution of critical points of potentials generated by an infinite discrete distribution of charge. This paper contains some preliminary results in this area and some new questions.

We would like to thank D. Drasin, A. A. Goldberg, W. K. Hayman and L. A. Rubel for helpful comments.

Received 9 July 1991; revised 15 January 1992.

1991 Mathematics Subject Classification 31 A05.

This research was carried out at the University of York while the third author was serving as a British Science and Engineering Research Council (SERC) fellow. He gratefully acknowledges the hospitality and support extended to him by the Department of Mathematics.

J. London Math. Soc. (2) 47 (1993) 309-320 


\section{Formulation of results}

If the $a_{j}$ are integers, we may consider the meromorphic function

$$
F(z)=\prod_{j=1}^{\infty}\left(1-\frac{z}{z_{j}}\right)^{a_{j}}
$$

which is of order at most one, minimal type. (It belongs to the convergence class of order 1). Then

$$
f(z)=\frac{F^{\prime}(z)}{F(z)} .
$$

If the $z_{j}$ are distinct and $a_{j}=1$ for all $j$ then (2.2) implies that $f$ has infinitely many zeros. Indeed in this case the zeros of $F^{\prime}$ are the zeros of $f$ and since $F^{\prime}$ is also of order at most one, minimal type, $F^{\prime}$ has infinitely many zeros.

THEOREM 2.1. If the $a_{j}$ are positive integers then $f$ has an infinite set of zeros.

This follows immediately from the following.

THEOREM 2.2. Let $F$ be a transcendental entire function of order at most one, minimal type. Then $F^{\prime} / F$ has an infinite set of zeros.

In the opposite direction we have

EXAMPLE 2.3. For every $\rho \geqslant 1$ there exists an entire function $F$ of order $\rho$ such that $F^{\prime} / F$ has no zeros.

A. A. Goldberg and W. K. Hayman brought the following result to our attention.

THEOREM 2.4. If $a_{j}= \pm 1$, then $f$ has an infinite set of zeros.

This is a consequence of a result of F. Nevanlinna [10]. Under the conditions of Theorem 2.4 the function $F$ defined by (2.1) has only simple zeros and poles. So if $f$ has a finite set of zeros, then $F$ has only a finite number of critical points. In [10] all functions of finite order with this property were described. They satisfy differential equations of the form

$$
\frac{F^{\prime \prime \prime}}{F^{\prime}}-\frac{3}{2}\left(\frac{F^{\prime \prime}}{F^{\prime}}\right)^{2}=R,
$$

where $R$ is a rational function. Asymptotic integration shows that such functions $F$ have normal type and order $n / 2$, where $n$ is an integer, $n \geqslant 2$.

If the $a_{j}$ are only supposed to be integers then $f$ may have no zeros. The simplest example is the following. Take

$$
F(z)=\tan ^{2} \sqrt{ } z
$$

which is a meromorphic function of order $1 / 2$, normal type. Then

$$
f(z)=\frac{F^{\prime}(z)}{F(z)}=\frac{2}{\sqrt{ } z \sin 2 \sqrt{ } z} \neq 0
$$

and $f$ has the form (1.1). A similar example was communicated to the authors by W. K. Hayman. This example has also come up in the work of S. Bank and I. Laine [1] on ordinary differential equations in the complex domain. 
EXAMPLE 2.5. For every $\rho \geqslant 1 / 2$ there exists a meromorphic function $F$ of order $\rho$ such that $F^{\prime} / F$ has no zeros.

On the other hand we have the following theorem

THEOREM 2.6. If $F$ is a transcendental meromorphic function of order at most $1 / 2$, minimal type then its logarithmic derivative $f$ has an infinite set of zeros.

Let us state some conjectures based on the above theorems and examples.

CONJECTURE 2.7. If the $a_{j}>0$ then the function (1.1) has zeros. Equivalently, subharmonic functions of the form (1.2) have critical points.

CONJECTURE 2.8. If the $a_{j}$ are real and

$$
\sum_{j:\left|z_{j}\right| \leqslant r}\left|a_{j}\right|=o(\sqrt{ } r)
$$

then the function (1.1) has zeros. Equivalently, $\delta$-subharmonic functions of the form (1.2) of order at most $1 / 2$, minimal type have critical points.

CONJECTURE 2.9. (W. K. Hayman) If the $a_{j}$ are integers and $a_{j} \geqslant-1$ then the function (1.1) has zeros.

It is fairly easy to see that Conjecture 2.9 is equivalent to the following simply stated problem: if $F$ is meromorphic of order at most one, convergence class, then $F^{\prime}$ has zeros. We remark that by Theorem 2.6 the problem is solved for order at most $1 / 2$, minimal type.

We can partially prove Conjecture 2.7 with the additional assumption (2.4).

THEOREM 2.10. ${ }^{1}$ Under the conditions $a_{j}>0$ and (2.4) the function (1.1) has an infinite set of zeros.

Theorem 2.10 may be compared with Keldysh's theorem [see 5, Chapter 5, Theorem 6.2] that if

$$
\sum_{j=1}^{\infty}\left|a_{j}\right|<\infty \text { and } \sum_{j=1}^{\infty} a_{j} \neq 0
$$

then the function (1.1) has zeros and moreover,

$$
\delta(0, f)=0 .
$$

Here $a_{f} \in \mathbb{C}$ and $\delta(0, f)$ is the usual Nevanlinna deficiency of $f$ at 0 .

Our proof of all mentioned results except Theorem 2.10 require some complex analysis. So in the space $\mathbb{R}^{m}, m \geqslant 3$, we are able to prove only the following weak result.

THEOREM 2.11. Let $x_{k} \in \mathbb{R}^{m}, m \geqslant 3$ be distinct, nonzero points, $x_{k} \rightarrow \infty$. Then the function

$$
u(x)=\sum_{k=1}^{\infty} \frac{a_{k}}{\left|x-x_{k}\right|^{m-2}}, \quad a_{k} \geqslant 1, \quad \sum_{k=1}^{\infty} \frac{a_{k}}{\left|x_{k}\right|^{m-2}}<\infty
$$

has an infinite set of critical points.

\footnotetext{
${ }^{1}$ Essentially the same theorem was proved independently by J. Miles.
} 
CONJECTURE 2.12. If $x_{k} \in \mathbb{R}^{m}$

then the function

$$
\sum_{k=1}^{\infty}\left|x_{k}\right|^{-1+m}<\infty
$$

$$
u(x)=\sum_{k=1}^{\infty}\left(\frac{1}{\left|x-x_{k}\right|^{m-2}}-\frac{1}{\left|x_{k}\right|^{m-2}}\right)
$$

has an infinite set of critical points.

\section{Preliminaries}

Let us begin by recalling some topological properties of a meromorphic function $f[8,11]$. Suppose $f\left(z_{0}\right)=w_{0}$ and $f^{\prime}\left(z_{0}\right)$ is nonzero and finite. Then we can analytically continue the branch of $f^{-1}$ taking $w_{0}$ to $z_{0}$ in a neighbourhood of $w_{0}$. There are two types of obstacles preventing a further continuation of $f^{-1}$. The first of these arises from critical points, that is points $z$ where $f^{\prime}(z)=0$. Here the point $f(z)$ is called a critical value. The second obstacle arises from asymptotic values of $f$. A point $a$ is called an asymptotic value if there exists a curve $\Gamma$ tending to infinity such that $f(z)$ tends to $a$ along $\Gamma$. If a domain $V$ contains no critical or asymptotic values then every branch of $f^{-1}$ can be analytically continued throughout $V$.

To classify the singularities of $f^{-1}$, fix $a \in \mathbb{C}$ and take a set $A$ of connected neighbourhoods of the point $a$ such that $\bigcap\{N: N \in A\}=a$. Then for every $N \in A$ choose a component $V_{N}$ of the preimage $f^{-1}(N)$ in such a way that $N_{1} \subset N_{2} \Rightarrow V_{N_{1}} \subset V_{N_{2}}$. There are two possibilities:

1. $\left\{\left\{V_{N}: N \in A\right\}\right.$ is a point $z \in \mathbb{C}$. This point may be non-critical or critical. In the latter case we say that $f^{-1}$ has an algebraic singularity (or algebraic branch point) over a.

2. $\bigcap\left\{V_{N}: N \in A\right]=\varnothing$. In this case we say that our choice $N \mapsto V_{N}$ defines a transcendental singularity of $f^{-1}$ over $a$. This transcendental singularity is called direct if some $V_{N_{0}}$ contains no preimages of the point $a$. (Then $V_{N}$ contains no preimages of $a$ for all $N \subset N_{0}$ ). If all $V_{N}$ contain preimages of $a$ then each of them contains an infinite number of these preimages. Such a singularity is called indirect. Note that $f^{-1}$ has a transcendental singularity over $a$ if and only if $a$ is an asymptotic value.

We need the following theorem.

Denjoy-CARLEMAN-Ahlfors TheOrem. Let $f$ be a meromorphic function and suppose that $f^{-1}$ has $n \geqslant 2$ direct transcendental singularities. Then

$$
\liminf _{r \rightarrow \infty} r^{-n / 2} T(r, f)>0
$$

If $f$ is entire (3.1) is equivalent to

$$
\underset{r \rightarrow \infty}{\lim _{r \rightarrow \infty} r^{-n / 2} \log M(r, f)>0 .}
$$

If an entire function $f$ has $n \geqslant 1$ distinct finite asymptotic values then (3.2) holds. 
We need also the following elementary proposition concerning isolated singularities of $f^{-1}$ (c.f. [11, p. 283]).

Proposition 3.1. Let $f$ be a meromorphic function in $\mathbb{C}$. Suppose there exists a point $a$ and a disk $N$ centred at a such that some branch of $f^{-1}$ can be analytically continued throughout $M \backslash\{a\}$. Let $V$ be the corresponding component of $f^{-1}(M \backslash\{a\})$. Then there are two possibilities.

(i) For some $z_{0}$ with $f\left(z_{0}\right)=a, V \cup\left\{z_{0}\right\}$ is a bounded Jordan domain, $f$ maps $n$ to 1 from $V$ to $M \backslash\{a\}$ and $f(\partial V)=\partial N$. (Note that $z_{0}$ may or may not be a critical point.)

(ii) $V$ is an unbounded, simply connected domain, and $\partial V$ is a simple curve which tends to $\infty$ in both directions. The point a has no preimages in $V$.

We note that if (ii) holds then $V$ is a neighbourhood of a special type of direct transcendental singularity called a logarithmic singularity or logarithmic branch point.

We shall use the notion of line complex $[11,5,12]$. Let us recall the main ideas here. Suppose that there exists a finite set $A=\left\{a_{1}, \ldots, a_{q}\right\}$ containing all critical and asymptotic values of $f$. It follows from Proposition 3.1 that all possible singularities of $f^{-1}$ are algebraic and logarithmic branch points. Consider a Jordan curve $J$ passing through the points $a_{1}, \ldots, a_{q}$. This divides the sphere $\overline{\mathbb{C}}$ into two components. Choose two points, one in each component and denote them by $\mathrm{X}$ and $\mathrm{O}$. The curve $J$ is divided by the points $a_{1}, \ldots, a_{q}$ into $q$ open arcs $l_{1}, \ldots, l_{q}$. For every $k$ connect the points $\mathrm{X}$ and $\mathrm{O}$ by a simple curve $\gamma_{k}$ such that the only point of intersection of $\gamma_{k}$ with $J$ lies on $l_{k}$, and all the $\gamma_{k}$ are disjoint. We get a graph $\gamma$ with two vertices $\mathbf{X}$ and $\mathbf{O}$ and $q$ edges $\gamma_{k}$. In the following picture $q=3$ and $a_{3}=\infty$.

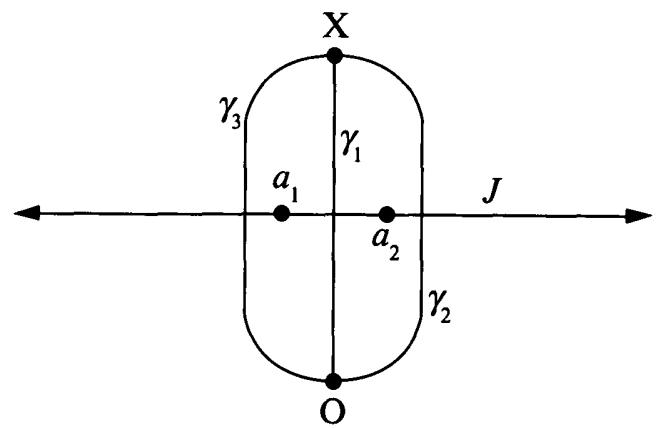

The full preimage $\Gamma=f^{-1}(\gamma) \subset \mathbb{C}$ is called a line complex (Streckenkomplexe) and has the following properties, which are almost evident.

1. $\Gamma$ is connected.

2. $\Gamma$ has vertices of two types: $X$ and $O$. Each edge connects two vertices of different types.

3. The number of edges adjacent to each vertex is equal to $q$.

It is known (and easy to prove using the Uniformization Theorem) that every graph with these properties embedded in the plane arises from some meromorphic function in the plane or in the unit disk having a finite set of critical and asymptotic values.

The faces of $\Gamma$ (which means connected components of $\mathbb{C} \backslash \Gamma$ ) are mapped to the neighbourhoods of the points $a_{k}$ (bounded by $\gamma_{k}$ and $\gamma_{k-1}$ ). So we may mark each face which is mapped to a neighbourhood of $a_{k}$ by the symbol $a_{k}$. It is easy to see that two faces marked by the same symbol have disjoint closures. Each face is bounded by an 
even number of edges. Each $2 n$-gonal face contains one $a_{k}$-point of multiplicity $n$. A logarithmic singularity corresponds to the face bounded by an infinite number of edges which forms the following simple chain.

$$
-\mathrm{O}-\mathrm{X}-\mathrm{O}-\mathrm{X}-\mathrm{O}-\mathrm{X}-\mathrm{O}-\mathrm{X}-\mathrm{O}-\mathrm{X}-
$$

Sometimes we shall consider a reduced line complex which means that we delete all 2-gons. A reduced line complex contains at most one edge connecting a pair of vertices.

\section{Proof of Theorem 2.2}

Suppose that $F^{\prime} / F$ has a finite set of zeros. So $F^{\prime}(z)=0 \Rightarrow F(z)=0$ with at most a finite number of exceptions. It follows that the set of critical values of $F$ is finite. By the Denjoy-Carleman-Ahlfors Theorem, the set of asymptotic values is also finite. Since $F$ is transcendental, Proposition 3.1 implies that there is a logarithmic branch point over $\infty$. Further since there are only finitely many finite singularities of $f^{-1}$, any finite asymptotic value is also a logarithmic branch point. Consequently by the Denjoy-Carleman-Ahlfors Theorem and the order restriction on $F, F$ can have no finite asymptotic value. That this is impossible follows from the following lemma with $a_{1}=0$.

LEMMA 4.1. Let $F$ be transcendental, entire with finitely many critical values $a_{1}, \ldots, a_{n}$. Suppose $E=\left\{z: F(z)=a_{i}, F^{\prime}(z)=0, i=2, \ldots, n\right\}$ is a finite set. Then $F$ has a finite asymptotic value.

Proof. If $E$ is empty, we get an immediate contradiction from Proposition 3.1 since it implies that $a_{1}$ is a logarithmic singularity and hence an asymptotic value. Suppose $F$ has no finite asymptotic value. Then there exists $R>0$ such that $U=\{z:|z|<R\}$ contains all the finite singularities of $F^{-1}$. Since any branch of $F^{-1}$ can be continued analytically without restriction in $\mathbb{C} \backslash \bar{U}$, it follows from Proposition 3.1 that any component $V$ of $F^{-1}(\mathbb{C} \backslash \bar{U})$ corresponds to a logarithmic singularity at infinity. Each component of $F^{-1}(\partial U)$ is a boundary component of such a domain $V$ and hence, in this case, coincides with $\partial V$. Thus $\partial V$ is a Jordan curve going to infinity in both directions. Fix a point $b$ in $U$ and insert $n$ line segments from $b$ to $\partial U$. This divides $U$ into $n$ disjoint regions $C_{1}, \ldots, C_{n}$ each bounded by consecutive rays, $R_{i}$ and $R_{i+1}$ (throughout, $n+1$ will be identified with 1 ). By moving $b$ if necessary we can arrange that $a_{i} \in C_{i}$.

By Proposition 3.1 and the assumption that $F$ has no finite asymptotic value, every component of $F^{-1}\left(C_{i}\right)$ is bounded and consequently, $F^{-1}\left(C_{i}\right)$ has infinitely many components for each $i, i=1, \ldots, n$. Since $E$ is finite, there exist components $B_{i}$ of $F^{-1}\left(C_{i}\right)$ such that $F^{\prime}$ is not zero in $\bar{B}_{i}, i=2, \ldots, n$ and $\partial B_{i} \cap \partial B_{i+1}$ maps into $R_{i+1}$. Thus, since there are no finite asymptotic values of $F$, the monodromy theorem gives that $F^{-1}$ is $1-1$ from $\overline{C_{2}} \cup \ldots \cup \overline{C_{n}}$ onto $\overline{B_{2}} \cup \ldots \cup \overline{B_{n}}$. Since the singularities of $F^{-1}$ in $\overline{C_{1}}$ lie over $a_{1}$, we can continue this branch of $F^{-1}$, to all of $U \backslash\left\{a_{1}\right\}$. Again by Proposition 3.1, the resulting component $V$ of $F^{-1}(U)$ is a bounded region with $F(\partial V)=\partial U$. But, as mentioned above, every component of $F^{-1}(\partial U)$ is a simple Jordan curve extending to infinity in both directions. This gives the desired contradiction. The lemma, and hence Theorem 2.2, is now proved. 
We present a more elegant (but essentially identical) proof of Lemma 4.1 using the notion of line complexes. Since our examples depend heavily on this notion, the two proofs may serve to help the reader better to understand the next section.

Consider the reduced line complex corresponding to $F$. It contains a face $D_{0}$ corresponding to the logarithmic singularity over $a_{q}=\infty$ which is bounded by a simple chain. The line complex also may contain a finite number of faces, say $D_{1}, \ldots, D_{n}$ marked by the points $a_{2}, \ldots, a_{q-1}$ and an infinite set of faces $D_{j}, j \geqslant n+1$, marked by $a_{1}=0$. Take two adjacent edges $w_{1}$ and $w_{2}$ on $\partial D_{0}$ which do not belong to the boundaries of $D_{1}, \ldots, D_{n}$. One of them, say $w_{1}$, does not belong to the boundary of any $D_{j}, j \geqslant n+1$, because the closures of $D_{j}, j \geqslant n+1$ are pairwise disjoint. So there is a face $D^{*}$ adjacent to $D_{0}$ along $w_{1}$ such that $D^{*}$ is not a $D_{j}, j \geqslant 0$. Since all faces corresponding to algebraic branch points are listed in the sequence $D_{j}, j \geqslant 1, D^{*}$ is a face corresponding to some finite logarithmic branch point other than that of $D_{0}$. This logarithmic branch point gives the required asymptotic value.

\section{Examples}

Our examples are based on the theory of Riemann surfaces with almost periodic ends which was developed by A. A. Goldberg [4] toward the solution of the inverse problem of Nevanlinna theory. An excellent self-contained presentation of the theory is in [5, Chapter 7].

Construction of Example 2.3. If $\rho=1$, we may take $F(z)=e^{z}$; so we suppose now that $\rho>1$. Fix a natural number $n \geqslant 2$ and consider the following two graphs called Block 1 and Block 2 . Block 1 contains $2 n$ vertices.<smiles>[X][X]O[X](C)OCO[Y]</smiles>

\section{BLock $1(n=4) \quad$ BLOCK 2}

Then fix a number $x, 0<x<1$ and find a sequence $\Sigma$ of natural numbers with the property

$$
\#(\Sigma \cap[1, m])=m x+O(1), \quad m \rightarrow \infty .
$$

Now construct the line complex $\Gamma$ as follows: take the sequence of blocks $\left(B_{k}\right)$, $-\infty<k<\infty$ such that

$$
\begin{aligned}
& B_{k} \text { is Block } 1 \text { if } k \in \Sigma, \\
& B_{k} \text { is Block } 2 \text { if } k \notin \Sigma .
\end{aligned}
$$

Then connect each $B_{k}$ to $B_{k+1}$ identifying the right free edge of $B_{k}$ to the left free edge of $B_{k+1}$. We get the following line complex when $n=4$ and $\Sigma=\{1,4,5, \ldots\}$.

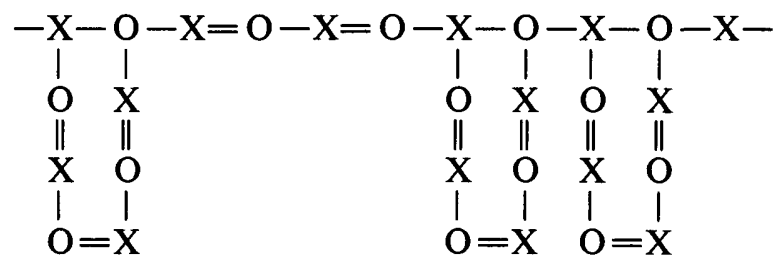


All conditions for this graph to be a line complex are satisfied. The corresponding function is ramified only over three points which we may choose to be 0,1 and $\infty$. Let us mark the upper infinite polygonal face by 1 , the lower one by $\infty$ and all the $2 n$-gonal faces by 0 .

It follows from the theory of almost periodic ends that the constructed line complex corresponds to a function $F$ meromorphic in $\mathbb{C}$ and having normal type of order

$$
\rho=1+\left(\frac{\log ((n-1) x+1)}{2 \pi}\right)^{2}
$$

[5, Theorem 7.1, (7.17)]. It is easy to see that one can satisfy (5.11) with given $\rho>1$ by choosing a natural number $n \geqslant 2$ and a positive $x<1$.

From the line complex we see that the function $F$ is entire since there are no faces with a finite number of edges marked by $\infty$. All critical points (which correspond to $2 n$-gonal faces) are mapped by $F$ to 0 . So our example has all the desired properties.

Construction of Example 2.5. Fix a natural number $n \geqslant 5$ and consider the following three blocks:

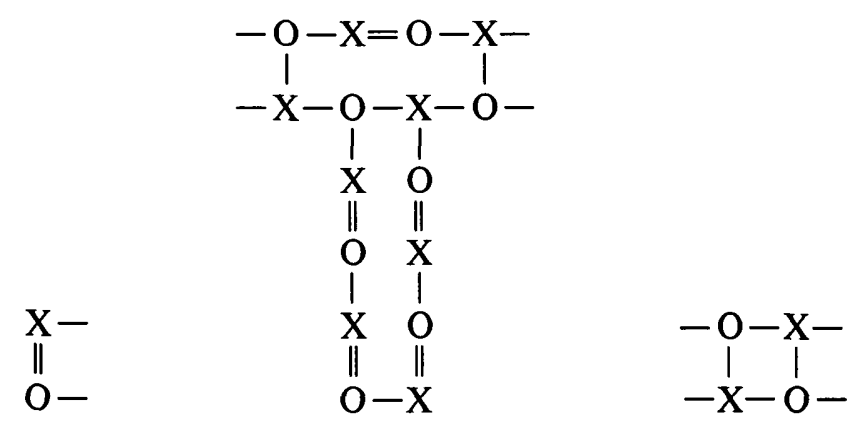

BLOCK $0 \quad$ BLOCK $1(n=8) \quad$ BLOCK 2

Block 1 has two faces: one of them is an 8-gon (above) and the other is a 2(n-3)-gon (below).

Fix a number $x, 0<x<1$ and choose a sequence of natural numbers $\Sigma$ satisfying (5.1). Then construct the line complex $\Gamma$ as follows: take a sequence of blocks $\left(B_{k}\right)$, $k=0,1,2, \ldots$ such that

$$
\begin{aligned}
& B_{0} \text { is Block } 0, \\
& B_{k} \text { is Block } 1 \text { if } k \in \Sigma, \\
& B_{k} \text { is Block } 2 \text { in all other cases. }
\end{aligned}
$$

Connect $B_{k}$ to $B_{k+1}$ by identifying the right pair of free edges of $B_{k}$ to the left pair of free edges of $B_{k+1}$. We get a line complex like this:

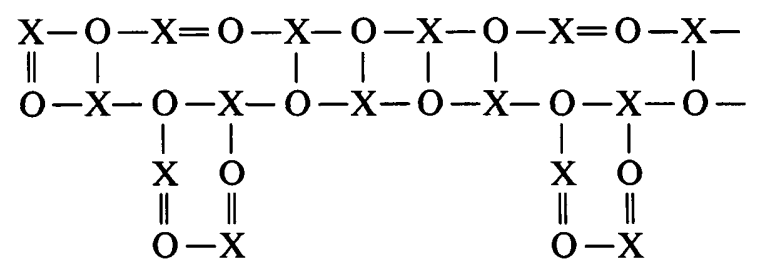


In the picture $n=6$ and $\Sigma=\{1,3, \ldots\}$. Mark all faces of $\Gamma$ which are polygons with a finite number of sides by 0 and $\infty$ such that adjacent faces have different markers. The only infinite face will be marked by 1 . It follows from the theory of almost periodic ends that there is a function $F$ meromorphic in $\mathbb{C}$ which corresponds to $\Gamma$. The only ramified values of $f$ are 0 and $\infty$. The order of $F$ is given by [5, Chapter 7 , (7.17)]:

$$
\rho=\frac{1}{2}\left(1+\left(\frac{1}{\pi} \log \left(\frac{(n-3) x+1}{x+1}\right)\right)^{2}\right) .
$$

It is easy to see that one can get an arbitrary order $\rho>1 / 2$ by choosing appropriate $n$ and $x$.

\section{Proof of Theorem 2.6}

We use a theorem by A. Huber [7] (see also [9] for another proof).

Huber's THEOREM. Let $g$ be a transcendental entire function. Then there exists a curve $\Gamma$ tending to infinity such that

$$
\int_{\Gamma}|g(z)|^{-1}|d z|<\infty
$$

Let us suppose that Theorem 2.6 is false and hence that $F^{\prime} / F$ has finitely many zeros. We write

$$
\frac{F^{\prime}}{F}=\frac{P}{g}
$$

where $P$ is a polynomial and $g$ is a transcendental entire function of order at most $\frac{1}{2}$, minimal type. We write $g=P_{1} g_{1}$ where $P_{1}$ is a polynomial of the same degree as $P$ and $g_{1}$ is entire. By Huber's Theorem applied to $g_{1}$, there exists a path $\Gamma$ from zero to infinity such that $F$ approaches a finite asymptotic value $a$ as $z \rightarrow \infty$ along $\Gamma$.

By the $\cos \pi \rho$-theorem [3] there exists a sequence of circles $\left\{z:|z|=r_{n}\right\}, r_{n} \rightarrow \infty$ such that

$$
\left|\frac{F^{\prime}}{F}\left(r_{n} e^{i \theta}\right)\right|=\left|\frac{P}{g}\left(r_{n} e^{i \theta}\right)\right| \rightarrow O\left(r_{n}^{-1}\right)
$$

uniformly in $\theta$ as $n \rightarrow \infty$. By (6.1) and the fact that $F(z) \rightarrow a$ as $z \rightarrow \infty, z \in \Gamma$, we have that

$$
F\left(r_{n} e^{i \theta}\right) \rightarrow a
$$

uniformly in $\theta$ as $r_{n} \rightarrow \infty$. Hence $F$ can have no other asymptotic value. Clearly then, we can find a Jordan neighbourhood $U$ of $a$ such that $F^{-1}$ has no singularities in $U \backslash\{a\}$. Thus $F$ has a logarithmic singularity over $a$. By (6.2) we have that $\left\{z:|z|=r_{n}\right\}$ is mapped into $U$ for large $n$. But Proposition 3.1 states that $F^{-1}(\partial U)$ is a curve tending to infinity in both directions, so we have a contradiction, which proves the theorem.

\section{Proof of Theorem 2.10}

The conditions of the theorem imply that the function $u$ given by (1.2) is subharmonic in $\mathbb{C}$ and has at most order $\frac{1}{2}$, minimal type. It follows from the $\cos \pi \rho$-theorem [3] that there exists a sequence $r_{k} \rightarrow \infty$ such that

$$
m\left(r_{k}\right):=\inf \left\{u(z):|z|=r_{k}\right\} \rightarrow \infty \text {. }
$$


Take an arbitrary natural number $N$ and fix $r_{0}$ such that at least $N$ of the points $z_{j}$ are contained in the disk $|z|<r_{0}$. Let

$$
M(r):=\max \{u(z):|z|=r\}
$$

Fix $k$ so large that

$$
m\left(r_{k}\right)>M\left(r_{0}\right)
$$

and consider the level set

$$
L(t)=\{z: u(z)<t\}, \quad-\infty<t \leqslant M\left(r_{0}\right) .
$$

If $t=M\left(r_{0}\right)$ then there is a component $L_{0}$ of the set $L(t)$ such that

$$
D\left(0, r_{0}\right) \subset L_{0} \subset D\left(0, r_{k}\right)
$$

Here $D(0, r)$ is the disk of radius $r$ centred at 0 . If $t$ is very small, the intersection $L(t) \cap L_{0}$ consists of at least $N$ simply connected components containing the points $z_{j}$. As $t$ increases these components may collide only at critical points of $u$. So $u$ has at least $N-1$ critical points in $D\left(0, r_{k}\right)$ counting multiplicity. As $N$ was chosen arbitrary large, the theorem is proved.

REMARK. Both Theorem 2.2 and Theorem 2.10 could have been proved a little more concisely (but with less of an elementary flavour) by using Huber's theorem. Indeed, we could obtain an alternate and immediate proof of Lemma 4.1 (with entire replaced by meromorphic!) giving a finite asymptotic value for $F$ in Theorem 2.2 which would lead to the same contradiction. In Theorem 2.10 Huber's Theorem gives a path $\Gamma$ on which the logarithmic potential $u$ is bounded. Since $u$ is subharmonic, a variant of the Denjoy-Carleman-Ahlfors Theorem shows that $u$ has order at least $\frac{1}{2}$, normal type, contradicting (2.4).

\section{Subharmonic functions in space. Proof of Theorem 2.11}

Under the conditions of this theorem $u$ is a non-negative superharmonic function. We shall essentially repeat the arguments of Section 7 based on (7.1). The only thing we need is a replacement of the $\cos \pi \rho$-theorem. We shall prove that the conditions of Theorem 2.11 imply that

$$
B(r):=\max \{u(x):|x|=r\} \longrightarrow 0
$$

on a sequence of $r$ tending to $\infty$.

REMARK. It is known [6] that positive superharmonic functions tend to zero outside a small exceptional set. But this does not imply (8.1). Indeed (8.1) may fail for a general positive superharmonic function, because the function may be equal to $\infty$ on a ray. As we shall see the crucial condition is $a_{k} \geqslant 1$.

We have

$$
B(r) \leqslant \sum_{k=1}^{\infty} \frac{a_{k}}{\left\|x|-| x_{k}\right\|^{m-2}}
$$

So all we need is the following lemma. 
LEMMA 8.1. Suppose that $0<x_{1}<x_{2}<\ldots, m \geqslant 3$ is an integer, $a_{k} \geqslant 1$ for all $k$ and

Set

$$
\sum_{k=1}^{\infty} a_{k} x_{k}^{2-m}<\infty
$$

Then

$$
\Psi(x)=\sum_{k=1}^{\infty} a_{k}\left|x-x_{k}\right|^{2-m} .
$$

$$
\liminf _{x \rightarrow+\infty} \Psi(x)=0 .
$$

Proof. We shall take $m=3$ for simplicity; the case $m>3$ can be treated similarly. Set

Then

$$
n(r)=\sum_{k: x_{k} \leqslant r} a_{k}
$$

$$
\sum_{k: x_{k} \leqslant r} \frac{a_{k}}{x_{k}}=\int_{0}^{r} \frac{d n(t)}{t}=\frac{n(r)}{r}+\int_{0}^{r} \frac{n(t) d t}{t^{2}} .
$$

It follows from (8.2) that

$$
\int_{0}^{\infty} \frac{n(t)}{t^{2}} d t<\infty
$$

So there exists a sequence $r_{j} \rightarrow \infty$ such that

Set

$$
n\left(r_{j}\right)=o\left(r_{j} / \log r_{j}\right), \quad j \longrightarrow \infty .
$$

and

$$
\phi(x)=\min \{1,1 /|x|\}
$$

$$
\Phi(x)=\sum_{k=1}^{\infty} a_{k} \phi\left(x-x_{k}\right) .
$$

Suppose that the lemma is not true. Then

$$
\liminf _{x \rightarrow+\infty} \Psi(x) \geqslant c
$$

for some $c, 0<c<1$. We deduce from (8.4) that

$$
\liminf _{x \rightarrow+\infty} \Phi(x) \geqslant c .
$$

Indeed, if dist $\left(x,\left\{x_{k}: k \in \mathbb{N}\right\}\right) \leqslant 1$ then $\Phi(x) \geqslant 1>c$, and if dist $\left(x,\left\{x_{k}: k \in \mathbb{N}\right\}\right)>1$ then $\Phi(x)=\Psi(x)$ so in either case we have $\Phi(x) \geqslant \min \{1, \Psi(x)\}$.

It follows from (8.5) that

for all sufficiently large $r$.

$$
\int_{0}^{r} \Phi(x) d x \geqslant \frac{1}{2} c r
$$

To obtain the desired contradiction we estimate this integral from above

$$
\begin{aligned}
\int_{0}^{r} \Phi(x) d x & =\sum_{k=1}^{\infty} a_{k} \int_{0}^{r} \phi\left(x-x_{k}\right) d x=\sum_{k=1}^{\infty} a_{k} \int_{-x_{k}}^{r-x_{k}} \phi(t) d t \\
& =\sum_{x_{k} \leqslant 2 r} a_{k} \int_{-x_{k}}^{r-x_{k}} \phi(t) d t+\sum_{x_{k}>2 r} a_{k} \int_{-x_{k}}^{r-x_{k}} \phi(t) d t \\
& =\Sigma_{1}+\Sigma_{2} .
\end{aligned}
$$


For $\Sigma_{1}$ and $\Sigma_{2}$ we have

$$
\begin{aligned}
\Sigma_{1} & \leqslant \sum_{x_{k} \leqslant 2 r} a_{k}\left(\log x_{k}+\log ^{+}\left(r-x_{k}\right)+2\right) \leqslant 2 n(2 r)(\log 2 r+2), \\
\Sigma_{2} & \leqslant \sum_{x_{k}>2 r} a_{k}\left(\log x_{k}-\log \left(x_{k}-r\right)\right)=\sum_{x_{k} \geqslant 2 r} a_{k} \log \left(1+\frac{r}{x_{k}-r}\right) \\
& \leqslant \sum_{x_{k} \geqslant 2 r} \frac{2 r a_{k}}{x_{k}}=o(r), \quad r \longrightarrow \infty .
\end{aligned}
$$

Putting this together and substituting $2 r=r_{j}$ where $r_{j}$ satisfies (8.3), we get

$$
\int_{0}^{r} \Phi(x) d x \leqslant 2 n\left(r_{j}\right)\left(\log r_{j}+2\right)+o\left(r_{j}\right)=o\left(r_{j}\right)=o(r),
$$

which contradicts (8.6).

\section{References}

1. S. BANK and I. Laine. 'On the oscillation theory of $f^{\prime \prime}+A f=0$ where $A$ is entire', Trans. Amer. Math. Soc. 273 (1986) 351-363.

2. D. BranNan and W. HAYMAN. 'Research problems in complex analysis', Bull. London Math. Soc. 21 (1989) $1-35$.

3. M. ESSEN. The $\cos \pi \rho$-theorem, Lecture Notes in Mathematics 467 (Springer, Berlin, 1975).

4. A. A. GolDBERG. 'Inverse problem of the value distribution theory of meromorphic functions', (Russian) Ukrainian Math. J. 6 (1954) 385-397.

5. A. A. Goldberg and I. V. Ostrovski. Distribution of values of meromorphic functions (Russian) (Nauka, Moscow, 1970).

6. W. K. Hayman and P. B. Kennedy. Subharmonic functions 1 (Academic Press, London, 1976).

7. A. HuBER. 'On subharmonic functions and differential geometry in the large', Comment. Math. Helv. 32 (1957) 13-72.

8. F. IVERSEN. Recherches sur les fonctions inverses des fonctions meromorphes. Thèse, University of Helsingfors 1914.

9. J. Lewis, J. Rossi and A. Weitsman. 'On the growth of subharmonic functions along paths', Ark. Mat. 22 (1983) 104-114.

10. F. Nevanlinna. 'Über eine Klasse meromorpher Funktionen', 7-le Congress Math. Scand. Oslo (1929) 81-83.

11. R. NevanlinNa. Analytic functions. (Translated by Philip Emery) Grundlehren der Mathematischen Wissenschaften 162 (Springer, Berlin, 1970).

12. H. WIттісH. Neuere Untersuchungen über eindeutige analytische Funktionen (Berlin, Springer, 1968).

(J.C.)

University of York

Heslington

York YO1 5DD

(J.R.)

Virginia Polytechnic Institute and State University

Blacksburg

VA 24061

USA
(A.E.)

University of Kentucky

Lexington

KY 40506-0027

USA

Current address:

Purdue University

West Lafayette

IN 47907

USA 